\title{
Factors affecting antibiotic decisions for upper respiratory tract infections I: A survey of family physicians
}

\author{
Dick E. Zoutman, ${ }^{1,2}$ B. Douglas Ford, ${ }^{1}$ Assil R. Bassili, Jarold L. Cosby, ${ }^{3}$ Kanji Nakatsu. ${ }^{4}$ \\ ${ }^{1}$ Department of Pathology and Molecular Medicine, Queen's University, Kingston, Ontario; \\ ${ }^{2}$ Infection Control Service, Kingston General Hospital, Kingston, Ontario; \\ ${ }^{3}$ Centre for Evaluation of Medicines, St. Joseph's Healthcare, Hamilton, Ontario; \\ ${ }^{4}$ Department of Pharmacology and Toxicology, Queen's University, Kingston, Ontario.
}

doi:10.3396/ijic.V4i1.005.08

\begin{abstract}
Identifying factors associated with the ubiquitous inappropriate prescribing of antibiotics for upper respiratory tract infections (URTIs) will help develop effective interventions and decrease antimicrobial resistance. Surveys were mailed to family physicians in Ontario, Canada. The survey assessed antibiotic prescribing for URTIs and a wide range of influences on antibiotic decisions. Multivariate models of inappropriate prescribing weregenerated. 316 of $544(58 \%)$ family physicians completed surveys. Associated with self-reported antibiotic prescribing for acute bronchitis were patients with obligations (OR 2.1; $95 \% \mathrm{Cl}, 1.2-3.6)$, physicians with positive antibiotic use attitudes (OR 2.1; 95\% Cl, 1.1-3.9), satisfaction antibiotics best for patients (OR 1.5; 95\% Cl, 1.1-2.1), and knowledgeable patients (OR 0.5; 95\% Cl, 03-0.8). Associated with antibiotic prescribing for influenza were patients with obligations (OR 2.2; $95 \% \mathrm{Cl}, 1.2-3.8$ ), patients thought to be seeking antibiotics (OR 1.4; $95 \%$ $\mathrm{Cl}, 1.1-1.9)$, and attending university and profession sponsored courses (OR 0.7; 95\% Cl, 0.4-1.0). Associated with not prescribing first line antibiotics for acute sinusitis were pharmaceutical industry influence (OR 2.0; $95 \% \mathrm{Cl}, 1.1-3.3$ ), solo practice (OR 2.0; $95 \% \mathrm{Cl}, 1.1-5.0$ ), and recommending rest and simple analgesics (OR 0.5; 95\% Cl, 0.3-0.8). Associated with not prescribing first line antibiotics for streptococcal pharyngitis were pharmaceutical industry influence $(\mathrm{OR} 1.7 ; 95 \% \mathrm{Cl}, 1.3-2.5)$, physician age (OR 1.6; 95\% Cl, 1.3-2.1), and perceived importance of clinical guidelines (OR 0.6; $95 \% \mathrm{Cl}, 0.4-0.8$ ). Health care workers should be informed of the influence of perceived patient motivation and the pharmaceutical industry on antibiotic use for URTIs and these insights included in interventions targeting inappropriate antibiotic prescribing.
\end{abstract}

\section{Corresponding author}

Dick E. Zoutman, MD, FRCPC, Department of Pathology and Molecular Medicine, Queen's University and Infection Control Service, Kingston General Hospital, 76 Stuart Street, Kingston, Ontario, K7L 2V7, Canada, Tel: 613 549-6666 X 4015, Fax: 613 548-2513, Email: zoutmand@kgh.kari.net 


\section{Background}

Throughout the globe antibiotics are inappropriately prescribed for upper respiratory tract infections (URTIs) in adults. ${ }^{1-7}$ The injudicious use of antibiotics contributes to the emergence of antimicrobial resistance, decreased therapeutic effectiveness of antibiotics, and increased health care costs. ${ }^{8}$ Decreased antibiotic use has been demonstrated to reduce antimicrobial resistance. ${ }^{9}$

Patient expectations are often cited as the reason for inappropriate antibiotic prescribing for URTIs. ${ }^{1014}$ Antibiotic prescribing practices for URTIs are also influenced by clinical error, ${ }^{15,16}$ diagnostic uncertainty, ${ }_{13}^{13,14}$ office location, ${ }^{17}$ workload, ${ }^{5,13,18}$ medico legal considerations, ${ }^{11,18}$ and method of remuneration. ${ }^{5}$

A survey of family physicians was conducted two years after province-wide dissemination of the Ontario Anti-infective Guidelines for Community-acquired Infections. ${ }^{19}$ Family physicians are responsible for the vast majority of community antibiotic prescriptions in Canada. We examined the influence of a wide range of factors on self-reported antibiotic prescribing practices for URTIs in adults. The identification of factors associated with inappropriate antibiotic prescribing will aid the development of effective interventions targeting antimicrobial resistance.

\section{Methods \\ Subjects}

Surveys were mailed to 544 family physicians in southeastern Ontario, Canada during the winter of 1999. Eligible physicians were not specialists in a discipline other than family medicine and did not limit their practice to one area (e.g. psychotherapy). A second survey was sent to non-responders.

\section{Instruments}

The survey covered a wide range of factors thought to influence antibiotic decisions for URTIs. The survey was pilot tested on six physicians experienced in family practice. Demographic information such as age, sex, and type of remuneration and practice information such as patient volume and office location was collected. Physicians were queried about the influence of treatment, practice, medical system, and patient factors on the prescribing of antibiotics, and perceptions of patients' motivation for consults and interactions with patients. Continuing medical education (CME) activities and non-antibiotic treatment practices for URTIs were also examined. Scenarios depicting the common cold, acute laryngitis, influenza, acute sinusitis, acute bronchitis, and streptococcal pharyngitis were presented to physicians who considered these scenarios in adult patients with no comorbidity or drug allergies and indicated whether they would prescribe an antibiotic. Next to each scenario were 15 choices: 13 commonly prescribed antibiotics, "Other, please specify" and "None". Physicians could choose more than one antibiotic for each syndrome.

\section{Dependent variables}

Antibiotics are not considered the standard of care for influenza and acute bronchitis in otherwise healthy adults. ${ }^{19}$ Physicians who reported prescribing antibiotics for the influenza and acute bronchitis scenarios in otherwise healthy adult patients were considered to be prescribing inappropriately for these indications. Antibiotics are considered the standard of care for acute sinusitis and streptococcal pharyngitis. ${ }^{19}$ Physicians were considered to be prescribing inappropriately for acute sinusitis and streptococcal pharyngitis if they did not report generally prescribing a first line antibiotic for these indications.

\section{Statistical analysis}

In order to assess the influence of a broad range of factors on prescribing practices, factor analysis was used to reduce the number of independent variables. Factor analysis is a data reduction technique that groups variables based on linear relationships. Identified factors are named to represent the survey items within the factor. The factor extraction method was principal components analysis with extracted factors having eigenvalues exceeding one and variables with factor loadings $\geq 0.5$ included in factor-based scales. ${ }^{20}$

Independent variables were tested for association with self-reported prescribing using univariate logistic regression. The independent variables included factorbased scales and 17 other independent variables direct from the survey including nine demographic and practice variables, three CME variables, an item about how well physicians knew patients, and four variables regarding patient motivations for visits 


\section{Table I. Independent variables tested for association with physician sel-reported prescribing practices}

\section{Indipendent variables derived from survey form}

Demographic \& Practice Information: Physician Age and Gender of Medical Training, Certification by the College of Family Physicians of Canada, Primary Type of Practice, Practice Location, Training Medical Students, Principal Source of Remuneration, \# of Patients Seen Daily

CME Participation: University sponsored and pharmaceutical company sponsored CME meetings attended. Whether physician meets with pharmaceutical sales representatives

How well physician knew patients

Physician Ascriptions of Patient's Motivation for Consults: Non-antibiotic treatment for symptom and or pain relief. Antibiotic prescription. Reassurance patient does not have more serious disease. Note to be off work or school

\section{Independent variables derived from factor analyses}

Treatment Factors: Physician Indicates desire to act. Prescribing to err on the side of caution. Positive antibiotic use attitudes

Practice \& Medical System Factors: Time and remuneration considerations. Lack of training in infectious diseases and antibiotics. Liability concerns, Interpersonal pressures to rescribe Patient Factors: Perceived patient expectations for antibiotics. Patient has obligations such as work and school, Adherence concerns, Co-morbiditiy concerns Non-antibiotic Treatment Recommendations: Rest and simple analgesics, Dietry Supplements, Over-the-counter medications, Antihistamines, Behavioural interventions Perceptions of and Interactions with Patients Factors: Knowledgeable patients and time to educate, Patient counseling, Physician satisfaction with prescribing antibiotics for URTIs Information Sources on Influence an Antibiotic Prescribing Factors: Clinical guidelines from medical sources, Pharmaceutical industry, Scientific medical literature, Professional and university-based meetings.

(Table I). Variables with univariate P-values $<0.05$ were tested by multiple logistic regression for inclusion in the multivariate model by stepwise logistic regression using forward selection if the significance level of the logistic likelihood ratio test was $<0.05$ with backward elimination if the significance level was $>0.05[21] .{ }^{21}$ Separate models were developed for the influenza, acute bronchitis, acute sinusitis, and streptococcal pharyngitis scenarios.

\section{Results}

\section{Respondent characteristics}

Surveys were received from 316 of 544 (58\%) physicians. The mean age was 46 (SD 11) and 65\% were male. The majority (64\%) were certified by the College of Family Physicians of Canada and $57 \%$ were in group practice, $39 \%$ in solo practice, and $4 \%$ in walk-in and community health clinics. The principal form of remuneration was fee-for-service (92\%) with salary for the remainder. Physicians reported seeing 29 (SD 10) patients per day during the winter and estimated 21\% (SD 15) of adult patients had URTIs and $27 \%$ (SD 19) of these patients received antibiotics.

\section{Factor analysis}

The factor analyses reduced 87 individual items in the survey to a manageable 23 factors. The factor analysis of 11 treatment-related items resulted in the identification of three treatment factors: physician desire to act, prescribing to err on the side of caution, and positive antibiotic use attitudes. Four factors were identified from the 15 practice and medical system-related questions: time and remuneration considerations, lack of training in infectious diseases, liability concerns, and interpersonal pressures to prescribe. The factor analysis of the 17 patient related items identified four factors: patient expectations for antibiotics, patient with obligations such as work and school, adherence concerns, and co-morbidity concerns. Five factors were derived from the 12 nonantibiotic recommendation-related items: rest and simple analgesics, dietary supplements, over-the- 
counter medications, antihistamines, and behavioral interventions. The factor analysis of the 16 patient interaction-related items resulted in the identification of three factors: perceived knowledge level of patients, patient counseling, and satisfaction with prescribing antibiotics. Four factors were derived from the 16 continuing medical education-related items: clinical guidelines from medical sources, pharmaceutical industry, scientific medical literature, and professional and university based meetings.

\section{Physician self-reported antibiotic prescribing for common URTIS}

Antibiotic prescribing for otherwise healthy adult patients without drug allergies for the common cold, acute laryngitis, influenza, acute bronchitis, acute sinusitis, and streptococcal pharyngitis scenarios are presented in Table II. Most physicians reported prescribing antibiotics for streptococcal pharyngitis $(99 \%)$ and acute sinusitis (97\%) and a third (35\%) reported prescribing antibiotics for acute bronchitis, a quarter $(23 \%)$ for influenza, $11 \%$ for acute laryngitis, and $4 \%$ for the common cold. A quarter of physicians
$(27 \%)$ reported not prescribing penicillin $\mathrm{V}$ the first line recommendation for streptococcal pharyngitis and $17 \%$ did not report prescribing amoxicillin and or co-trimoxazole the first line recommendations for acute sinusitis.

\section{Factors affecting antibiotic decisions for non-antibiotic requiring URTIs}

There was sufficient variability in self-reported antibiotic prescribing for the acutebronchitis and influenzascenarios to test the association of the independent variables listed in Table 1 with these indications. The multivariate model of antibiotic prescribing for acute bronchitis contained four factors. Patients with obligations such as work, school, and travel were reported to be more likely to receive antibiotics for acute bronchitis (OR 2.1; 95\% Cl, 1.2-3.6; $\mathrm{P}=0.008$ ). Physicians indicating positive antibiotic use attitudes, such as antibiotics are relatively harmless and effective for URTIs, were more likely to report prescribing antibiotics for acute bronchitis (OR 2.1; 95\% Cl, 1.1-3.9; $\mathrm{P}=0.02$ ). In a similar vein, physicians who indicated they were satisfied they had done the best for the patient when they prescribed antibiotics for URTIs, were more

Table II. Physician self-reported prescribing of antibiotics for common upper respiratory tract infections

\begin{tabular}{|c|c|c|c|c|c|c|}
\hline $\begin{array}{l}\text { Antibiotic } \\
\text { Prescribed (\%)* }\end{array}$ & $\begin{array}{r}\text { Strptococcal } \\
\text { Pharyngitis }\end{array}$ & $\begin{array}{r}\text { Acute } \\
\text { Sinusitis }\end{array}$ & $\begin{array}{r}\text { Acute } \\
\text { Bronchitis }\end{array}$ & Influenza & $\begin{array}{r}\text { Acute } \\
\text { Laryngitis }\end{array}$ & $\begin{array}{r}\text { Common } \\
\text { Cold }\end{array}$ \\
\hline Physicians & 99 & 97 & 35 & 23 & 11 & 4 \\
\hline \multicolumn{7}{|c|}{ Prescribing Antibiotics } \\
\hline Amoxicillin** & 46 & 65 & 20 & 8 & 5 & 3 \\
\hline Amoxicillin- & 6 & 28 & 2 & 0 & 0 & 1 \\
\hline \multicolumn{7}{|l|}{ Clavulinate } \\
\hline Penicillin V & 73 & 1 & 0 & 3 & 1 & 0 \\
\hline Cephalexin & 4 & 7 & 2 & 0 & 0 & 0 \\
\hline $2^{\text {nd }}$ Generation & 5 & 19 & 4 & 1 & 0 & 0 \\
\hline \multicolumn{7}{|l|}{ Cephalosporinst † } \\
\hline Fluoroquinolones & 1 & 5 & 2 & 1 & 0 & 0 \\
\hline Erythromycin & 30 & 13 & 19 & 11 & 5 & 0 \\
\hline Extended Spectrum & 9 & 31 & 12 & 13 & 3 & 0 \\
\hline \multicolumn{7}{|l|}{ Macrolides ‡ } \\
\hline Tetracycline & 2 & 4 & 4 & 2 & 1 & 0 \\
\hline \multicolumn{7}{|l|}{ Co-trimoxazole } \\
\hline $\begin{array}{l}\text { *Physicians could prescri } \\
\text { ** Amoxicillin and related } \\
\text { + Cefaclor, Cefuroxime; } \\
\text { \# Clarithromycin and Azi }\end{array}$ & $\begin{array}{l}\text { ore than one ant } \\
\text { oo-penicillins; } \\
\text { ycin }\end{array}$ & & & & & \\
\hline
\end{tabular}


likely to report prescribing antibiotics for acute bronchitis (OR 1.5; 95\% Cl, 1.1-2.1; $\mathrm{P}=0.02$ ). When physicians perceived patients as more knowledgeable, knowing URTIs generally resolved on their own and physicians had the time to educate patients, physicians reported they prescribed antibiotics less often (OR $0.5 ; 95 \% \mathrm{Cl}$, 03-0.8; $\mathrm{P}=0.006$ ).

The multivariate model of antibiotic prescribing for influenza contained three factors. Physicians reported that patients with obligations such as work, school, and travel were more likely to be prescribed antibiotics for influenza (OR 2.2; 95\% Cl, 1.2-3.8; $\mathrm{P}=0.008$ ). When physicians thought patients were motivated to consult in order to receive antibiotics, physicians reported prescribing antibiotics for influenza more often (OR 1.4; 95\% Cl, 1.1-1.9; $\mathrm{P}=0.01$ ). Physicians attending more university and professionally sponsored courses and symposia reported they prescribed antibiotics less often for influenza (OR 0.7; 95\% Cl, 0.4-1.0; $\mathrm{P}=0.04)$.

\section{Factors affecting antibiotic decisions}

for antibiotic requiring URTIS

The independent variables listed in Table 1 were tested for association with not prescribing first line antibiotic choices by logistic regression for acute sinusitis and streptococcal pharyngitis. The multivariate model for acute sinusitis contained three factors. Physicians who ascribed greater influence to pharmaceutical industry information sources such as advertisements in medical journals, sales representatives, industry educational materials, and pharmaceutical companysponsored meetings reported they were more likely not to prescribe first line antibiotics for acute sinusitis (OR 2.0; 95\% Cl, 1.1-3.3; $\mathrm{P}=0.02$ ). Physicians in solo practices reported prescribing first line antibiotics less often than did physicians in group practices (OR 2.0; 95\% Cl, 1.1-5.0; $\mathrm{P}=0.02$ ). Physicians more likely to recommend rest and simple analgesics reported prescribing first line antibiotics more appropriately (OR 0.5; 95\% Cl, 0.3-0.8; $\mathrm{P}=0.002$ ).

The multivariate model for streptococcal pharyngitis contained three factors. Physicians who ascribed greater influence to pharmaceutical industry information sources (OR 1.7; 95\% Cl, 1.3-2.5; $\mathrm{P}=0.03$ ) were more likely not to report prescribing first line antibiotic choices. Whereas physicians who ascribed greater influence to clinical guidelines from medical sources (OR 0.6; 95\% Cl, 0.4-0.8; $\mathrm{P}=0.0009$ ) prescribed more appropriately. Older physicians were more likely to not report prescribing first line antibiotics for streptococcal pharyngitis (OR 1.6; 95\% Cl, 1.3-2.1; $\mathrm{P}=0.0002)$.

\section{Physician comments}

Almost all who offered comments (266 of 271) indicated antibiotic prescribing could be decreased for URTIs without lowering standards of care. The most frequent responses to the question about how antibiotic prescribing could be reduced were public/ patient education (64\%), physician education (39\%), better remuneration for patient education $(7 \%)$, faster in office diagnostic tests and laboratory results (4\%), and assertiveness in dealing with patient demands (4\%).

The most frequent response when asked about influences on the prescribing of antibiotics for URTIs was patient expectations (39\%). This was followed by the belief that walk-in clinics "herd" patients through and cater to patient demand for antibiotics (22\%). Patient co-morbidity was mentioned by $14 \%$ of physicians and $14 \%$ also listed business concerns such as fee structures that reward prescribing and fears that patients will transfer. The pharmaceutical industry was seen by $6 \%$ as influencing the prescribing of antibiotics for URTIs. Only two physicians mentioned physician knowledge deficits.

\section{Discussion}

A sizeable minority of physicians reported generally prescribing antibiotics for otherwise healthy patients with non-antibiotic requiring URTIs. While only a small percentage of reported prescribing antibiotics for the common cold and acute laryngitis, a third reported prescribing antibiotics for acute bronchitis and a quarter for influenza. Antibiotics are recommended for streptococcal pharyngitis and acute sinusitis and a substantial minority of physicians reported not prescribing first line antibiotics for these indications.

Physician perceptions of patient desire for antibiotics includes perceived patient antibiotic knowledge levels, patients having obligations, and patient expectations for antibiotics were associated with self-reports of prescribing antibiotics for both acute bronchitis and 
influenza. This is consistent with the literature linking perceived patient expectations with prescribing antibiotics against indication for URTIs. ${ }^{10-14}$ Physician perceptions of patient expectations for antibiotics; however, have been shown to be inaccurate. ${ }^{12}$ There is a need to examine what patients expect from consults for URTIs and to inform health care workers of what patients expect.

Physicians with positive attitudes towards the use of antibiotics and physicians that attended fewer university and profession sponsored CME were at risk for prescribing antibiotics for non-antibiotic requiring indications. Physician knowledge and education factors have previously been identified as influences on the prescribing of antibiotics for URTIs. ${ }^{15,16,18}$ Only two physicians in our survey listed physician knowledge deficits as a factor in prescribing antibiotics for URTIs; somewhat paradoxically, physician education was often mentioned as a strategy to reduce antibiotic prescribing. The effectiveness of educational interventions in improving antibiotic prescribing for URTIs further suggests the integral role of physician knowledge. ${ }^{4,7,22,23}$

An implication of our findings is the need for educational interventions that target the treatment of URTIs, especially with regard to indications for antibiotic treatment and antibiotic drug choices. Profession and university-sponsored initiatives would be preferable to pharmaceutical industry-sponsored initiatives, as industry contact was associated with inappropriate prescribing in our study and other studies. ${ }^{24}$

A limitation of our study was that it was based on selfreported prescribing. Physicians might be motivated to present their prescribing practices in the best light although this did not appear to be the case. The use of conservative modeling procedures increased the possibility of excluding some potential factors from the models while decreasing the possibility of including false positives. ${ }^{21}$

Our examination of a wide range of variables identified a number of factors that influence antibiotic decisions for URTIs. Perceptions of patient desire for antibiotics were associated with self-reported prescribing for acute bronchitis and influenza. When antibiotics were required, physicians who ascribed greater influence to pharmaceutical industry sources were less likely to report prescribing first line antibiotics. This study provides insights into the widespread injudicious prescribing of antibiotics for URTIs. These insights can help design interventions to improve antibiotic practices. A natural extension of this study would be to examine the influences of a broad range of variables on actual antibiotic prescribing.

\section{Acknowledgements}

Research supported by The Physicians' Services Incorporated Foundation, 5160 Yonge Street, Suite 1006, Toronto, Ontario, Canada, M2N 6L9

\section{References}

1. Zoutman DE, Ford BD, Bassili AR. Antibiotic prescribing by family physicians for upper respiratory tract infections: impacts of feedback, non-antibiotic drug recommendations, and patient factors. International Journal of Infection Control 2006: 2: 65-71.

2. du Prey B, Ford BD, Bassili AR, Zoutman DE. Antimicrobial recommendations by health care providers in Kenya. Am I Health Syst Pharm. 2004; 61: 302-304.

3. McGregor A, Dovey S, Tilyard M. Antibiotic use in upper respiratory tract infections in New Zealand. Fam Pract. 1995; 12: $166-170$.

4. Perez-Cuevas R, Guiscafre $\mathrm{H}$, Munoz $\mathrm{O}$, et al. Improving physician prescribing patterns to treat rhinopharyngitis. Intervention strategies in two health systems of Mexico. Soc Sci Med. 1996; 42: 1185-1194.

5. Hutchinson JM, Foley RN. Method of physician remuneration and rates of antibiotic prescription. CMAJ. 1999; 160: 10131017.

6. Mazzaglia G, Greco S, Lando C, Cucinotta G, Caputi AP. Adult acute upper respiratory tract infections in Sicily: pattern of antibiotic drug prescription in primary care. I Antimicrob Chemother. 1998; 41: 259-266.

7. Gonzales R, Malone DC, Maselli JH, Sande MA. Excessive antibiotic use for acute respiratory infections in the United States. Clin Infect Dis. 2001; 33: 757-762.

8. Conly J. Antimicrobial resistance in Canada. CMAJ 2002; 167: 885-891.

9. Seppala H, Klaukka T, Vuopio-Varkila J, Muotiala A, Helenius $H$, Lager K, Huovinen P. The effect of changes in the consumption of macrolides antibiotics on erythromycin resistance in group A streptococci in Finland. N Engl / Med. 1997; 337: 491-492.

10. Britten N, Ukoumunne O. The influence of patients' hopes of receiving a prescription on doctors' perceptions and the decision to prescribe: a questionnaire survey. BMJ. 1997; 315: 1506-1510.

11. Lam TP, Lam KF. What are the non-biomedical reasons which make family doctors over-prescribe antibiotics for upper respiratory tract infection in a mixed private/public Asian setting? J Clin Pharm Ther. 2003; 28: 197-201.

12. Hamm RM, Hicks RJ, Bemben DA. Antibiotics and respiratory infections: are patients more satisfied when expectations are met? J Fam Pract.1996; 43: 56-62. 
13. Bradley CP. Uncomfortable prescribing decisions: a critical incident study. BMJ.1992; 304: 294-296.

14. Miller E, MacKeigan LD, Rosser W, Marshman J. Effects of perceived patient demand on prescribing anti-infective drugs. CMAJ. 1999; 161: 139-142.

15. Gonzales R, Barrett PH Jr, Steiner JF. The relation between purulent manifestations and antibiotic treatment of upper respiratory tract infections. / Gen Intern Med. 1999; 14: 151 156.

16. Mclsaac WI, Butler CC. Does clinical error contribute to unnecessary Antibiotic use? Med Decis Making. 2000; 20: 3338.

17. Mainous AG 3d, Hueston WJ, Clark JR. Antibiotics and upper respiratory infection: do some folks think there is a cure for the common cold. J Fam Pract. 1996; 42: 357-361.

18. Butler CC, Rollnick S, Pill R, Maggs-Rapport F, Stott N. Understanding the culture of prescribing: qualitative study of general practitioners' and patients' perceptions of antibiotics for sore throats. BMJ. 1998; 317: 637-642.
19. Ontario Anti-infective Review Panel. Anti-Infective Guidelines for Community-Acquired Infections. Toronto: Queen's Printer for Ontario; 1997.

20. Hutcheson G, Sofroniou N. The Multivariate Social Scientist. Thousand Oaks, CA: Sage; 1999.

21. Hosmer DW, Lemeshow S. Applied Logistic Regression. Toronto: Wiley; 1989.

22. Zwar N, Wolk J, Gordon J, Sanson-Fisher R, Kehoe L. Influencing antibiotic prescribing in general practice: a trial of prescriber feedback and management guidelines. Fam Pract. 1999; 16: 495-500.

23. Gonzales R, SteinerJF, LumA, BarrettPHJr. Decreasing antibiotic use in ambulatory practice: impact of a multidimensional intervention on the treatment of uncomplicated acute bronchitis in adults. JAMA.1999; 281: 1512-1519.

24. Wazana A. Physicians and the pharmaceutical industry: is a gift ever just a gift? JAMA. 2000; 283: 373-380. 